\title{
Gamificación aplicada al desarrollo del interés del alumnado por el contenido de una asignatura del Grado en Derecho
}

\author{
Gamification Applied to the Development of Students' \\ interest for the Content of a Subject of the Degree in Law
}

\author{
DIANA CAROLINA WISNER GLUSKO \\ Centro de Estudios Universitarios Cardenal Spínola CEU \\ (D) https://orcid.org/0000-0003-2723-6712
}

Recibido: 04/09/20

Aceptado: 02/12/20

\section{RESUMEN}

En los últimos años, la gamificación -el uso de juegos y técnicas de diseño de juegos en contextos que no son juegos- se ha ido asentando como una metodología innovadora que mejora la calidad educativa en la Educación Superior. La educación es un área de gran potencial para la aplicación de la gamificación. Dependiendo del nivel formativo, el docente debe establecer un momento concreto para aplicarla dentro del proceso de enseñanza-aprendizaje y también escoger un tipo de herramienta para diseñar y desarrollar en el aula. Este estudio ofrece una experiencia de gamificación basada en breakout para la enseñanza del Derecho Administrativo en el Grado en Derecho, y aplicada el primer día de clase, con una triple finalidad: promover la interacción entre los alumnos, medir el grado de conocimiento sobre los contenidos y las competencias adquiridas el pasado curso académico y motivar a los estudiantes haciendo más atractiva la enseñanza de esta materia.

\begin{abstract}
In recent years, gamification - the use of games and game design techniques in non-game contexts- has established itself as an innovative methodology that improves educational quality in Higher Education. Education is an area of great potential for the application of gamification. Depending on the educational level, the teacher must establish a specific moment to apply it within the teaching-learning process and also choose a type of tool to design and develop in the classroom. This study offers a gamification experience based on breakout for the teaching of Administrative Law in the Degree in Law, and applied on the first day of class, with a triple purpose: to promote interaction between students, to measure the degree of knowledge about the content and skills acquired in the last academic year and motivate students by making the teaching of this subject more attractive.
\end{abstract}

PALABRAS CLAVES Gamificación, Breakout Educativo, Innovación Educativa, Universidad, Derecho Administrativo, Motivación.

\section{KEYWORDS}

Gamification, BreakoutEDU, Educational Innovation, University, Administrative Law, Motivation
Para citar este artícullo: Wisner-Glusko, D. C. (2020). Gamificación aplicada al desarrollo del interés del alumnado por el contenido de una asignatura del grado en derecho. EA, Escuela Abierta, 23, 83-91. doi:10.29257/EA23.2020.06 


\section{INTRODUCCIÓN}

Al finalizar el curso académico, los profesores de todas las etapas educativas nos enfrentamos al gran desafío de planificar, de forma innovadora, las asignaturas para el próximo curso.

Innovar - del latín innovāre- significa, según la RAE, mudar o alterar algo introduciendo novedades. Por tanto, se entiende la innovación, en términos generales, como algo nuevo, como una aportación singular a un espacio determinado (Pérez, 2009); y, en concreto, la innovación en el ámbito educativo se podría designar tanto a una actividad por la que se conduce el proceso para la incorporación de algo nuevo en la institución escolar, como el resultado de la misma e implica los conceptos asociados de renovación, reforma y mejora escolar (Navarro-Asencio, 2017). Aunque señala Barraza-Macías (2005) que la valoración de lo que es innovador o no, está condicionado por diversos factores como las concepciones ideológicas, educativas y epistemológicas acerca de la educación y el proceso de enseñanza-aprendizaje.

En el marco del Espacio Europeo de Educación Superior (EEES) el concepto de enseñanza impulsa a utilizar métodos de aprendizaje más activos y orientados al estudiante universitario. Por tanto, como los alumnos son el centro del modelo educativo, no solo es necesario un cambio metodológico en la enseñanza, sino también que el docente utilice herramientas innovadoras y nuevas estrategias didácticas que ayuden a fomentar la participación del alumnado y aumente su motivación (De Soto, 2018). Y, para utilizar dichas herramientas, resulta fundamental que el profesorado posea las competencias y habilidades necesarias para ello, además del componente motivacional y actitudinal, requisitos indispensables en el docente innovador.

Hace exactamente un año, los docentes de todos los Grados del Centro de Estudios Universitarios "Cardenal Spínola" CEU (Adscrito a la Universidad de Sevilla) tuvimos la oportunidad de participar en el I Seminario de Gamificación en Entornos Educativos, organizado por nuestro Centro. Esta acción formativa se realizó con el objetivo de dar a conocer y reflexionar sobre las potencialidades y límites de la gamificación de los procesos educativos, a través del diseño práctico de actividades para el aula.

Precisamente del Taller que posteriormente realizamos, como actividad práctica del Seminario, surgieron las bases del diseño de un breakout, la experiencia de gamificación objeto del presente artículo.

La experiencia que aquí se expone tiene como principal objetivo evaluar, a partir de las percepciones de los estudiantes del Tercer Curso del Grado en Derecho, una experiencia de gamificación que se ha desarrollado a través de un breakout educativo con la finalidad de lograr que los educandos apliquen conceptos adquiridos en el curso anterior, desarrollen y profundicen nuevas habilidades y competencias educativas, incrementen su motivación y además que facilite la cohesión de grupo, todo ello en el marco de la presentación de la asignatura Derecho Administrativo II, al inicio del cuatrimestre.

\section{GAMIFICACIÓN EN LA ENSEÑANZA SUPERIOR}

La gamificación es una metodología innovadora, o como lo definen Parra-González y Segura-Robles (2019), es una metodología emergente, que consiste en utilizar juegos o mecánica de juego en contextos no lúdicos, proporcionando en educación la motivación en los alumnos. Mientras que Kapp (2012) y Zichermann y Cunningham (2011) hacen hincapié, para definirla, en los objeticos que persigue la utilización de la estética del juego y sus estrategias, y que no son otros que involucrar a la gente, motivar la acción, promover el aprendizaje y resolver problemas. 
Para Werbach y Hunter (2014) gamificación es una palabra engorrosa y que no captura todos los elementos del fenómeno, aunque señalan que deberían tomarse en serio los juegos, por el grado de implicación que produce en quienes participan, por las posibilidades de experimentación que ofrece y por los resultados que se obtienen al aplicarlo.

Las mecánicas de los juegos, como destacan Cortizo-Pérez, Carrero-García, Monsalve-Piqueras, Velasco-Collado, Díaz del Dedo y Pérez-Martín (2011), presentan una concatenación de reglas que permiten que los jugadores disfruten y generen compromisos mediante diferentes retos, y un camino por el que se pueda avanzar hasta una meta. También puede ser una herramienta útil para favorecer una participación de mayor calidad al generar vínculos emocionales positivos, fruto de sus experiencias (Martín-Padilla, 2018).

La gamificación es un concepto cada vez más presente en distintos ámbitos de nuestras vidas, aplicándose a entonos empresariales (ventas, marketing, gestión de personas, etc.) como en educación, saludo, gobierno, o incluso en nuestras tareas cotidianas (Teixes, 2015).

También llamada ludificación, estos últimos años ha visto incrementada su utilización en la enseñanza Superior, tanto en la Formación Profesional (FP) de grado medio para el aprendizaje de asignaturas relacionadas con Ciencias de la Tierra (De Soto-García, 2018), con la Formación y Orientación Laboral (Pérez-Rueda, 2018), en el Ciclo Formativo de Grado Superior en Integración Social (Manzano-León, Sánchez-Sánchez, Trigueros-Ramos, Álvarez-Hernández y Aguilar-Parra, 2020) o de Alojamientos Turísticos (Del Cerro-Gómez, 2015); como en la formación Universitaria y en materias muy diversas: didáctica del Deporte (Benítez-Porres, 2015), principios de las Relaciones Públicas (Rodríguez-Fernández, 2017), inglés (Pérez-Fernández, 2018), marketing (Piqueras-Casado y Alarcón del Álamo, 2019) o fisiología humana (Mauricio, Serna y Vallés, 2015). Y específicamente en el Área de conocimiento del Derecho, donde podría evidenciarse una cierta dificultad en aplicar la gamificación al proceso de enseñanza-aprendizaje, por el contrario, se reconoce como una herramienta de apoyo a la docencia (Sánchez-Frías, 2019).

Nadie puede desconocer que más del 50\% del éxito en una gamificación depende del diseño del juego, de la estrategia que se plantee aplicar. Teixes (2015) expone cuatro modelos para el diseño de un sistema gamificado, elaborando finalmente su propia propuesta y que consiste en las siguientes fases:

$1^{\circ}$ Definir los objetivos, considerando las razones por la que aplicar la gamificación.

$2^{\circ}$ Entender la audiencia objetiva y analizar el contexto

$3^{\circ}$ Delinear conductas objetivo, es decir los comportamientos que queremos inducir en los usuarios/ jugadores para que nos ayuden a obtener los objetivos planteados.

$4^{\circ}$ Definir los elementos necesarios para construir el sistema gamificado.

$5^{\circ}$ Implementarlo y ponerlo en funcionamiento.

$6^{\circ}$ Mantener la integración actualizada, en función de los resultados obtenidos (métricas).

Teniendo en cuenta todos estos elementos, a continuación, expondremos el porqué de esta experiencia de gamificación (objetivos), la metodología elegida para plantear la ludificación en el aula, las acciones realizadas tanto para el diseño como para su implementación y los resultados obtenidos. 


\section{OBJETIVOS DE LA GAMIIFICACIÓN}

Utilizando la psicología del juego, con esta actividad al inicio del primer día de clases, se han perseguido los siguientes objetivos específicos:

En primer lugar, que los alumnos recordaran y consolidaran conceptos del curso anterior necesarios para la asignatura de Derecho Administrativo correspondiente al nuevo Curso Académico. En segundo lugar, lograr que los estudiantes se sintieran motivados e implicados con la materia desde el inicio del nuevo curso, mejorando su desempeño académico mediante el uso del juego. En tercer término, propiciar una mayor integración de los alumnos de nuevo ingreso con los antiguos alumnos, a través del trabajo colaborativo y en equipo.

Finalmente, que esta experiencia permitiera a la profesora conocer y evaluar el estado de profundización del conocimiento sobre las instituciones jurídicas más importantes de Derecho Administrativo I, asignatura del curso anterior, cuyos conceptos básicos y conocimientos adquiridos sobre el procedimiento administrativo son esenciales para facilitar el proceso de enseñanza-aprendizaje de la asignatura del curso superior.

\section{DISEÑO Y METODOLOGÍA DE UN BREAKOUT EN DERECHO ADMINISTRATIVO}

A continuación, se describe la metodología utilizada, los participantes, los materiales y la aplicación de la estrategia de gamificación diseñada.

\subsection{Metodología}

Con la finalidad de alcanzar los objetivos y poner en marcha un proceso que incremente la motivación de los estudiantes que se refleje en una actitud comprometida con su formación, dentro de la metodología de gamificación, se ha optado por la aplicación del breakout.

Se trata de un juego inmersivo derivado del escape room, cuyo objetivo es abrir una caja, cofre o sobre cerrado. Para ello es necesario resolver cuestionarios, enigmas, crucigramas y realizar pruebas de conocimiento y deducción, desarrollando diferentes habilidades y competencias, incluso algunas vinculadas a los contenidos curriculares.

Negre i Walczak (2017) señala una serie de razones que fundamentan la aplicación del breakout en el aula: 1a) Su adaptación a cualquier contenido curricular y por tanto a cualquier asignatura; $2^{\text {a) }}$ Promociona la colaboración y el trabajo en equipo; $3^{\text {a }}$ ) Facilita el desarrollo del pensamiento crítico y la habilidad para resolver problemas; 4a) Contribuye a mejorar la competencia comunicativa; $5^{\text {a }}$ Plantea retos a los alumnos ante los que deben perseverar, (6a) Ayuda en la construcción del pensamiento deductivo; 7a) Los estudiantes aprenden a trabajar bajo presión; 8a) Los alumnos son los verdaderos protagonistas del aprendizaje; y 9a) El componente lúdico que favorece la participación e incrementa la motivación.

De esta manera se procedió a diseñar el breakout con motivo de la presentación de la asignatura. La idea principal ha sido que lo estudiantes descubrieran por sí mismo los temas principales que conforman el programa de estudio de la materia y dispusieran del manual básico de la asignatura en el aula, teniendo en cuenta el poder educativo del juego, la necesidad de contar con un alto grado de implicación de los estudiantes y la consecución de los objetivos propuestos. 


\subsection{Participantes}

Han participado de la experiencia un total de 31 estudiantes del Tercer Curso del Grado en Derecho, del Centro de Estudios Universitarios "Cardenal Spínola" CEU (adscrito a la Universidad de Sevilla), matriculados en la asignatura obligatoria Derecho Administrativo II, correspondiente al primer cuatrimestre del Curso Académico 2020/2021. De ellos han sido 16 mujeres y 15 hombres.

Más del 90\% de los alumnos que han intervenido se habían matriculado de la asignatura Derecho Administrativo I. Este dato ha sido muy importante a la hora de evaluar la adquisición de contenidos sobre las instituciones básicas del Derecho Administrativo, a fin de ser tenidas en cuenta para futuras acciones formativas del grupo.

\subsection{Materiales, diseño e implementación}

En el marco de las mecánicas del juego, se realizaron las siguientes actividades:

\section{a) Formación de Grupos y simulación}

- División de la clase en nueve grupos, conforme a la cantidad de temas del Programa de la asignatura.

- Identificación de los alumnos con un broche con el logo de uno de los Despacho más prestigiosos de España (elaborados por la profesora).

- Agrupación en las mesas identificadas con el mismo logo, simulando ser abogados de dichos bufetes.

\section{b) Introducción}

- Narrativa: "Un importante cliente quiere escoger al mejor Despacho de abogados para sus asuntos vinculados al Derecho Administrativo. Para ello os va a poner a prueba sobre los conocimientos adquiridos el pasado año en esta materia. Por grupos debéis ir sorteando una serie de retos y resolver enigmas hasta lograr descubrir dos palabras que tendréis que escribir en la tabla de la pizarra. Cada grupo tendrá un espacio concreto en esa tabla; y será el ganador quien las complete de forma correcta y en el menor tiempo posible. Es importante que la tabla esté totalmente rellena por lo que cada grupo recibirá una puntuación según el orden de finalización de la actividad. Dicha puntuación contará como primera calificación de la evaluación continua. Disponéis de un único comodín para preguntar a la profesora”.

\section{c) Resolución del primer enigma}

- Análisis de los elementos de un acto administrativo (elementos). Descubrimiento de una serie de números para poder abrir un sobre. 


\section{d) Resolución del segundo enigma}

- Apertura del sobre con una serie de instrucciones para descubrir las dos palabras.

- Resolución del crucigrama (confeccionado por la docente con preguntas de todos los temas del programa de la asignatura del curso anterior), y obtención de la primera palabra.

\section{e) Resolución del tercer enigma}

- Utilización de una hoja de papel en blanco con un orificio que estaba dentro del sobre. La pista es: "Encontraréis la palabra en una lista de materiales, capítulos o nombres contenidos en un libro o publicación junto con el número de páginas. ¿A dónde iríais a buscarlo dentro de nuestro Centro de Estudios Universitarios?”

- Visita de los alumnos a la biblioteca en busca del libro de la nueva asignatura para descubrir la segunda palabra.

\section{f) Finalización del juego}

- Obtención de las dos palabras y relleno de la tabla en la pizarra.

- Determinación de los ganadores y de las calificaciones obtenidas para cada grupo.

\section{g) Presentación de la asignatura por parte de la profesora,}

así como del programa de estudios utilizando la tabla confeccionada por los alumnos durante la realización del juego, de la normativa a utilizar y la bibliografía (con los ejemplares que han solicitado en biblioteca).

\section{RESULTADOS Y VALORACIONES DEL PROYECTO}

Medir el éxito de cualquier actividad de innovación docente es fundamental para valorar la consecución de los objetivos propuestos. Por esta razón, al finalizar la experiencia de gamificación, se realizó una encuesta de satisfacción entre los alumnos participantes. La misma recogía 11 preguntas, con respuesta de si/no para las vinculadas a experiencias anteriores de los alumnos sobre gamificación y, con una escala de puntuación de 1 a 5 (siendo 5 "si, mucho" y 1 "no, nada) para valorar la propia experiencia en el aula.

El desarrollo de la estrategia basada en el breakout, que aquí se describe, ha arrojado unos resultados muy positivos.

Para la totalidad de los alumnos encuestados el juego les ha ayudado a participar en clase. El 80\% ha valorado la experiencia de gamificación con la mejor calificación (5); y más del 80\% ha valorado con 4 y 5 que la realización del juego los ha motivado para afrontar la nueva asignatura con mayor entusiasmo. Para el 90\% de los encuestados la realización de este juego les ha ayudado a recordar y aplicar conceptos del año anterior. 
En esa misma línea, tanto el componente lúdico del breakout como el componente competitivo entre los compañeros, han sido decisivos a la hora de incentivar la participación del alumnado.

Finalmente, más del 80\% de los estudiantes manifestaron no solo que les ha gustado la experiencia de gamificación, sino que les gustaría que se repitiera en la asignatura Derecho Administrativo II así como en otras asignaturas del Grado.

Desde la perspectiva del docente, valoramos de forma significativa que la totalidad de los grupos superaron todos los retos y finalizaron con éxito la actividad, siendo que el 95\% de los alumnos nunca había participado en una experiencia de gamificación en sus estudios de Derecho.

También es importante resaltar que las respuestas de los grupos de estudiantes, a cada uno de los retos planteados en el breakout, ha proporcionado una valiosa información a la profesora sobre el nivel de solvencia en la aplicación de los conocimientos adquiridos el curso académico anterior en este área del Derecho Público y que son esenciales para la adquisición de nuevos conceptos vinculados a la asignatura de nuevo curso.

\section{CONCLUSIONES}

El desarrollo y aplicación de las dinámicas propias del juego en la enseñanza del Derecho Administrativo han sido altamente positivos para la consecución de los objetivos propuestos. Ello supone una motivación para una futura utilización de la gamificación en el proceso de enseñanza-aprendizaje de la asignatura.

La estrategia de gamificación planteada ha tenido dos pilares fundamentales en su diseño de cara al alumno: "aprender haciendo" (learning by doing) al momento de analizar el contenido del texto jurídico y resolver todos los enigmas y crucigramas y "aprender interactuando" (learning by interacting), porque el trabajo colaborativo ha sido fundamental para avanzar en el juego.

Los resultados obtenidos de las encuestas avalan el logro de los objetivos planteados. Ha quedado demostrado, por un lado, que la gamificación promueve la participación del alumnado y su deseo de aprender y, por otro, que es muy importante reforzar el trabajo en equipo y de forma colaborativa desde el primer día de clase.

Bien es cierto que la gamificación no asegura el aprendizaje -como no lo asegura ninguna metodología-, ni tiene como finalidad hacer divertida la clase en sí misma. No obstante, si los alumnos realizan una buena inmersión en el juego propuesto, lograrán trabajar con su motivación intrínseca (personal) y con su motivación extrínseca (como grupo), contribuyendo a lograr una mayor implicación del alumnado en el proceso de enseñanza- aprendizaje de la asignatura.

Sería interesante repetir la experiencia los sucesivos cursos académicos para poder comprobar si, efectivamente, el breakout es una herramienta metodológica que motiva a los alumnos e influye positivamente en el rendimiento académico de los educandos.

La innovación docente solo puede ir de la mano de buenas prácticas educativas, como lo es la gamificación, basadas en principios pedagógicos sólidos que estimulen y fomenten la motivación, la participación y la implicación responsable del alumnado en su formación universitaria. 


\section{REFERENCIAS BIBLIOGRÁFICAS}

Barraza-Macías, A. (2005). Una conceptualización comprehensiva de la innovación educativa. Innovación Educativa, 5(28), 19-31. Recuperado el 15/06/2015 de: https://www.redalyc.org/pdf/1794/179421470003.pdf.

Benítez-Porres, J. (2015). Socrative como herramienta para la integración de contenidos en la asignatura "Didáctica de los Deportes". En XII Jornadas Internacionales de Innovación Universitaria, Villaviciosa de Odón, 20 y 21 de julio, 2015. Recuperado el 20/07/2020 de http://abacus.universidadeuropea.es/handle/11268/4513

Cortizo-Pérez, J. C., Carrero-García, F., Monsalve-Piqueras, B., Velasco-Collado, A., Díaz del Dedo, L. y PérezMartín, J. (2011) Gamificación y Docencia: Lo que la Universidad tiene que aprender de los Videojuegos. En M. J. García-García y E. Icarán (Coords.), VIII Jornadas Internacionales de Innovación Universitaria Retos y oportunidades del desarrollo de los nuevos títulos en educación superior. Recuperado el 07/07/2020 de: https:// abacus.universidadeuropea.es/bitstream/handle/11268/1750/46_Gamificacion.pdf

De Soto-García, I. S. (2018). Herramientas de gamificación para el aprendizaje de ciencias de la tierra. Edutec. Revista Electrónica de Tecnología Educativa, 65, 29-39. doi: https://doi.org/10.21556/edutec.2018.65.1143

Del Cerro-Gómez, G. (2015). Aprender jugando, resolviendo: diseñando experiencias positivas de aprendizaje. En XII Jornadas Internacionales de Innovación Universitaria Educar para transformar: Aprendizaje experiencial. Recuperado el 20/05/2020 de: http://hdl.handle.net/11268/4334

Kapp, K. M. (2012). The Gamification of Learning and Instruction: Game-based Methods and Strategies for Training and Education. San Francisco: John Wiley.

Martín-Padilla, A. H. (2018). La Gamificación como estrategia motivadora hacia el aprendizaje en la Universidad. En E. López-Meneses, D. Cobos-Sanchiz, M. Padilla, A. Hilario, L. Molina-García y A. Jaén-Martínez (Coords.), Experiencias pedagógicas e innovación educativa. Aportaciones desde la praxis docente e investigadora. (pp. 21352149). Octaedro. Recuperado el 08/07/2020 de: http://hdl.handle.net/10433/6411

Mauricio, M., Serna, E. y Vallés, S (2015). Experiencias en la aplicación de la gamificación en $1^{\circ}$ Curso de Grado de Ciencias de la Salud. En Congreso In-Red 2015 Universitat Politècnica de València doi: http://dx.doi. org/10.4995/INRED2015.2015.1583

Manzano-León, A., Sánchez-Sánchez, M., Trigueros-Ramos, R., Álvarez-Hernández, J., y Aguilar-Parra, J. M. (2020). Gamificación y Breakout Edu en Formación Profesional. El programa «Grey Place» en Integración Social. EDMETIC, Revista de Educación Mediática y TIC, 9(1), 1-20. DOI: https://doi.org/10.21071/edmetic. v9i1.12067

Navarro-Asencio, E. (Coord.) (2017). Fundamentos de la investigación y la innovación educativa. Universidad Internacional de la Rioja.

NegreiWalczak, C.(2017). “BreakoutEdu”, microgamificación y aprendizaje significativo.educaweb.com. Recuperado el 22/07/2020 de https://www.educaweb.com/noticia/2017/07/26/breakoutedu-microgamificacionaprendizaje-significativo-15068/

Parra-González, Ma E. y Segura-Robles, A. (2019). Producción científica sobre gamificación en educación: un análisis cienciométrico. Revista de Educación, 386, 113-135.

Pérez, R. (2009). Planificación y diseño de programas para la innovación de centros formativos. En A. Medina (Ed.). Innovación de la Educación y de la docencia (pp. 121-163). Madrid: Ramón Areces.

Pérez-Fernández, L. (2018). El Escape room como herramienta de gamificación en la clase de inglés: una experiencia 
con alumnos universitarios. En E. López-Meneses, D. Cobos-Sanchiz, M. Padilla, A. Hilario, L. MolinaGarcía; A. Jaén-Martínez, Experiencias pedagógicas e innovación educativa. Aportaciones desde la praxis docente e investigadora (pp.448-458) Octaedro. Recuperado el 25/07/2020 de: http://hdl.handle.net/10433/6411

Pérez-Rueda, M. B. (2017). Gamificación, una propuesta metodológica para las aulas de FP (Tesis de Maestría) Universitat Jaume I, Valencia. Recuperado el 18/07/2020 de: http://hdl.handle.net/10234/174105

Piqueras-Casado, L. y Alarcón del Álamo, M. (2019). Gamificación y estilos de aprendizaje en el Grado en Administración y Dirección de Empresas de la Universidad de Murcia. En L. J. Belmonte-Ureña, J. J. GázquezLinares, M. Simón-Márquez y col. (Eds.), Innovación Docente e Investigación en Ciencias Sociales, Económicas y Jurídicas (pp.431-440). Madrid: Dykinson.

Rodríguez-Fernández, L. (2017). Smartphones y aprendizaje: el uso de Kahoot en el aula universitaria. Revista Mediterránea de Comunicación/Mediterranean Journal of Communication, 8(1), 181-190. Recuperado de: https:// www.mediterranea-comunicacion.org/article/view/2017-v8-n1-smartphones-y-aprendizaje-el-uso-dekahoot-en-el-aula-universitaria

Sánchez-Frías, (2019). La gamificación como herramienta de apoyo a la docencia. En L. J. Belmonte-Ureña, J. J. Gázquez-Linares, M. Simón-Márquez et al. (Eds.), Innovación Docente e Investigación en Ciencias Sociales, Económicas y Jurídicas (pp.559-565). Madrid: Dykinson.

Teixes, F. (2015). Gamificación: fundamentos y aplicaciones. Barcelona: Editorial UOC.

Werbach, K. y Hunter D. (2014). Gamificación. Revoluciona tu negocio con las técnicas de los juegos. Madrid: Pearson Ecucation.

Zichermann, G. y Cunningham, C. (2011). Gamification by Design: Implementing Game Mechanics in Web and Mobile Apps. Cambridge: O’Reilly Media.

\section{INFORMACIÓN SOBRE LA AUTORA}

Diana Carolina Wisner-Glusko. Profesora Titular del Centro Cardenal Spínola CEU (adscrito a la Universidad de Sevilla) de Derecho Administrativo y Derecho de las Telecomunicaciones y las Nuevas Tecnologías. Gestora del Área de Conocimiento del Grado en Derecho. Doctora en Derecho por la Universidad Carlos III de Madrid (2009). Magister en Finanzas Públicas, provinciales y municipales por la Facultad de Economía de la Universidad Nacional de La Plata. Licenciada en Derecho por la Universidad de Sevilla (Homologación de Título Extranjero) y Abogada por la Facultad de Ciencias Jurídicas y Sociales de la Universidad Nacional de La Plata. Maestra de Educación Básica. Sus intereses de investigación son la digitalización de las Administraciones Públicas, la administración electrónica, la accesibilidad de los sitios web de los organismos del sector público, el Derecho de las Nuevas Tecnologías y la innovación didáctica en el Grado en Derecho. 